\title{
How often do primary care veterinarians record the overweight status of dogs?*
}

\author{
Nicola C. Rolph ${ }^{1}$, Peter-John M. Noble ${ }^{1}$ and Alexander J. German ${ }^{2 \dagger}$ \\ ${ }^{1}$ School of Veterinary Science, University of Liverpool, Leaburst Campus, Chester High Road, Neston, Wirral CH64 7TE, UK \\ ${ }^{2}$ Department of Obesity and Endocrinology, University of Liverpool, Leahurst Campus, Chester High Road, Neston, Wirral CH64 7 TE, UK
}

(Received 7 November 2013 - Final revision received 14 March 2014 - Accepted 21 March 2014)

Journal of Nutritional Science (2014), vol. 3, e58, page 1 of 5

doi:10.1017/jns.2014.42

Abstract

Obesity is a prevalent medical condition in dogs caused by the excess accumulation of fat, with negative effects on quality of life, longevity and the risk of developing associated pathologies. However, it is unclear how frequently first-opinion veterinarians record dogs as overweight (OW) or obese in medical records, and what factors determine when they do. Data sourced through the Small Animal Surveillance Network were used to determine the relative frequency of recording OW status (obesity or OW) in dogs presented to the UK first-opinion practices. Cases were identified using a search of clinical record-free text for relevant keywords. A case-control study was then conducted, comparing dogs where the OW status was recorded with a control group of obese dogs with no diagnosis recorded. Of 49488 consultations, the OW status was recorded in 671 dogs (relative frequency $1.4 \%$ ). Using multiple logistic regression, the OW status of a dog was more likely to be recorded when the consultation was for osteoarthritis (OR 5.42; $95 \%$ CI 2.09, 14.07; $P<0.001$ ) or lameness (OR 2.02; $95 \%$ CI 1.20, 3.42; $P=0.006$ ). Furthermore, the OW status was more commonly recorded in dogs that were members of a practice health scheme (OR 5.35; $95 \%$ CI 1.57, 18.17; $P=0.04)$ and less commonly recorded in microchipped dogs $(\mathrm{OR}$ $0.43 ; 95 \%$ CI $0.41,0.91 ; P=0.02)$. These results suggest that $\mathrm{OW}$ and obesity are underdiagnosed in the first-opinion practice. However, a presentation for orthopaedic disease appears a key prompt for recording the OW status. Further studies are now warranted to determine the reasons for such marked underdiagnosis.

Key words: Obesity: Canine nutrition: Practice surveillance

Obesity is defined as a disease in which excess body fat has accumulated such that health may be adversely affected ${ }^{(1)}$, and is one of the most common medical diseases in $\operatorname{dogs}(2,3)$. Recent UK studies put the prevalence of overweight (OW) and obese dogs between $52^{(3)}$ and $59 \%{ }^{(4)}$, with a rising trend and worldwide problem suggested ${ }^{(2,4)}$.

Owners commonly misperceive the body shape of dogs, and this is the most marked for OW $\operatorname{dogs}{ }^{(5-7)}$. Thus, it is vital for veterinarians to provide guidance as to what constitutes ideal body condition, and support this with effective education and communication regarding obesity prevention ${ }^{(8)}$. Despite this, a previous study demonstrated that veterinarians rarely perform body condition scores (BCS) on dogs in primary care practice, suggesting that they rarely discuss the issue of OW status with owners. There are a number of possible reasons for this, including more pressing medical problems ${ }^{(9)}$, the fact that the owner is obese ${ }^{(10)}$ or time limitations ${ }^{(11)}$.

The main aim of the present study was to determine the frequency of veterinarians recording dogs as obese or OW in the first-opinion practice. The study used a larger, more representative population of dogs than previous studies, and utilised data obtained from veterinary practice management software. The aim of a further study was to identify factors that were associated with the likelihood of OW status being recorded.

Abbreviations: BCS, body condition score; NSAID, non-steroidal anti-inflammatory drug; OW, overweight.

†Corresponding author: Alexander J. German, fax +44151795 6101, email ajgerman@liv.ac.uk

* This article was published a part of the WALTHAM International Nutritional Sciences Symposium Proceeding 2013. 


\section{Experimental methods}

This was a retrospective, correlational case-control study. Data were collected from practices using a compatible version of practice management software (Premvet v03.02.12) following a positive response to a postal request. seventy-four practices were approached, recruiting 16/59, 3/7, 0/6 and $0 / 2$ practices in England, Wales, Scotland and Northern Ireland, respectively (total nineteen practices comprising forty-two premises). Data were collected between 10 May 2010 and 8 August 2011. Data were stored in, and retrieved from, a relational database (MySQL, Oracle). In total, 73000 small animal consultations were contained within the database, of which 49 488 related to dogs.

All client-identifying data (e.g. owner name, address, postcodes and practice details) were either removed or coded to maintain anonymity of participants. Information was then exported to a spreadsheet application (Microsoft Excel version 10.6871.6870, Microsoft Corporation) and included signalment data such as breed, date of birth, sex, microchipping and insurance status, as well as free text written by the veterinarian about the consultations and the date conducted. Consultation duplicates were identified and removed prior to data analysis.

Cases (e.g. consultations where the dog was identified as having an OW status (i.e. the text included a term that suggested the dog was either obese or OW)) were selected from the total 49488 canine consultations by a search of the clinical record free text using words or phrases that correspond to likely diagnoses by veterinarians (Supplementary data). The number of initial case consultations identified was used to calculate the relative frequency of recording the OW status in dogs within the Small Animal Surveillance Network database. Consultations with missing information on date of birth, consultation date, breed, sex and neuter status were then excluded, since these variables were required for matching purposes. Furthermore, consultations with mixed-breeds and animals less than 2 years of age were excluded, since variance in weight within these categories might have affected valid selection of control animals. The free text for each consultation was then assessed, in order to confirm all details, and 231 consultations remained.

A matched group of controls (e.g. consultations where the dog was likely to be OW or obese, but this was not stated in the free text (i.e. none of the search terms were present)) was also identified). Given that BCS was recorded in $<25 \%$ of dogs, it was not possible to use this measure to identify OW or obese control dogs. Instead, OW control dogs were identified using body weight data: for each case, all similar dogs (e.g. with the same breed, age, sex and neuter status) were first identified in the database, and those with a body weight in the upper quartile of weight within this group were then selected (calculated by a formula in Microsoft Excel). For each case, three control dogs were selected from this group using the random series generator of a statistical software package (StatsDirect, version 2.6.8, StatsDirect Ltd.). If it was not possible to match a case to three controls, the case was removed. This method ensured that control dogs were matched to cases for breed and sex, and were among the heaviest dogs with the same signalment. Ultimately, there were 146 remaining cases and 438 matched controls. Signalment factors (age, sex, neuter status, breed (i.e. all breeds with more than ten dogs in the case group)) and body weight were compared between groups to confirm adequacy of group matching.

For all cases and controls, additional variables recorded were: vaccination appointment; parisiticide prescribed (including both endo- and ecto-parasiticides); non-steroidal anti-inflammatory drug (NSAID) prescribed; People's Dispensary for Sick Animals (a charity subsidising veterinary care for people receiving housing benefit or local government tax) registered; pet health club member; osteoarthritis present; lameness present; vomiting noted; diarrhoea noted; anal sacs expressed; aggressive behaviour noted; and abdominal palpation noted. Consultations were already defined in the database as 'yes' or 'no' for the further variables of microchipping and whether or not the animal was insured. The absolute frequencies of each variable for both cases and controls were recorded, and their relative frequencies calculated. Data were then exported to a statistical software package (StatsDirect, version 2.6.8) and binary logistic regression was used to calculate $\mathrm{OR}$ and $P$ values for each variable. Initially, simple regression was conducted. Thereafter, a multiple regression model was constructed, which initially included all variables that were $P \leq 0 \cdot 20$. The model was subsequently refined by backwards-stepwise elimination of the least significant variable at each round. Variables were retained in the final model, either if they were significant in their own right, or if removal lead to a significant $(>10 \%)$ change in the effect of the other variables. The level of statistical significance was set at $P<0 \cdot 05$, for two-sided analyses.

\section{Results}

\section{Relative frequency of veterinarians recording overweight} status

Based upon the search terms, the OW status of a dog was recorded in 671 of 49488 consultations, a relative frequency of $1.4 \%$.

\section{Case and control group summaries}

In the case group, median age was 7 years (range $2-15$ years), and this was similar to the age of controls (median 7 years, range 2-16 years). In both groups, $49 \%$ were male (cases 72/146; controls 216/438) and $51 \%$ were female (cases 74/ 146 , controls $222 / 438$ ), while $75 \%$ were neutered in both groups (cases 110/146, controls 330/438). In cases, median weight was $25.3 \mathrm{~kg}$ (range $4 \cdot 1-57 \cdot 0 \mathrm{~kg}$ ), while the median weight of control dogs was $24.9 \mathrm{~kg}$ (range $4 \cdot 1-54.4 \mathrm{~kg}$ ). A total of twenty-two breeds were included, with Labrador Retrievers (thirty-three, $23 \%$ ), Jack Russell Terriers (twentyone, $14 \%$ ), Staffordshire Bull Terriers (fifteen, $10 \%$ ), border collies (eleven, $8 \%$ ) and West Highland white terriers (ten, $7 \%$ ) contributing the most consultations. 
Table 1. Simple and multiple logistical regression analysis on factors associated with a veterinarian recording overweight status

\begin{tabular}{|c|c|c|c|c|c|}
\hline & & Cases (146) & Controls (438) & OR $(95 \% \mathrm{Cl})$ & $P$ value \\
\hline \multicolumn{6}{|l|}{ Simple regression } \\
\hline Age (years) ${ }^{\star}$ & & 7 years ( $2-16$ years) & 7 years ( $2-12$ years) & $0.99(0.94,1.05)$ & 0.799 \\
\hline \multirow{2}{*}{ Sex } & Female & $74(51 \%)$ & $222(51 \%)$ & (Reference level) & - \\
\hline & Male & $72(49 \%)$ & $216(49 \%)$ & $1.00(0.69,1.45)$ & $>0.999$ \\
\hline \multirow[t]{2}{*}{ Neuter status } & Entire & $36(25 \%)$ & $108(25 \%)$ & (Reference level) & - \\
\hline & Neutered & $110(75 \%)$ & $438(75 \%)$ & $1.00(0.65,1.54)$ & $>0.999$ \\
\hline \multirow[t]{2}{*}{ Labrador } & No & $113(77 \%)$ & $99(77 \%)$ & (Reference level) & - \\
\hline & Yes & $33(23 \%)$ & $339(23 \%)$ & $1.00(0.64,1.56)$ & 0.998 \\
\hline \multirow{2}{*}{ JRT } & No & $125(86 \%)$ & $375(86 \%)$ & (Reference level) & - \\
\hline & Yes & $21(14 \%)$ & $63(14 \%)$ & $7.37(0.00, \infty)$ & $>0.999$ \\
\hline \multirow[t]{2}{*}{ SBT } & No & $131(90 \%)$ & $393(90 \%)$ & (Reference level) & - \\
\hline & Yes & $15(10 \%)$ & $45(10 \%)$ & $1.00(0.54,1.85)$ & $>0.999$ \\
\hline \multirow[t]{2}{*}{ Border collie } & No & $135(92 \%)$ & $405(92 \%)$ & (Reference level) & - \\
\hline & Yes & $11(8 \%)$ & $33(8 \%)$ & $1.00(0.49,2.03)$ & $>0.999$ \\
\hline \multirow[t]{2}{*}{ WHWT } & No & 136 (93 \%) & $408(93 \%)$ & (Reference level) & - \\
\hline & Yes & $10(7 \%)$ & $30(7 \%)$ & $1.00(0.48,2.10)$ & $>0.999$ \\
\hline \multirow[t]{2}{*}{ Vaccinated } & No & $135(92 \%)$ & $409(93 \%)$ & (Reference level) & - \\
\hline & Yes & $11(8 \%)$ & $29(7 \%)$ & $1.15(0.56,2.37)$ & 0.705 \\
\hline \multirow[t]{2}{*}{ Insured } & No & $103(71 \%)$ & $275(63 \%)$ & (Reference level) & - \\
\hline & Yes & $43(29 \%)$ & $163(37 \%)$ & $0.70(0.47,1.06)$ & 0.090 \\
\hline \multirow{2}{*}{ Microchipped } & No & $87(60 \%)$ & $212(48 \%)$ & (Reference level) & - \\
\hline & Yes & $59(40 \%)$ & 226 (52 \%) & $0.64(0.44,0.93)$ & 0.020 \\
\hline \multirow[t]{2}{*}{ Practice health scheme } & No & $140(98 \%)$ & $433(96 \%)$ & (Reference level) & - \\
\hline & Yes & $6(2 \%)$ & $5(4 \%)$ & $3.71(1.12,12 \cdot 35)$ & 0.032 \\
\hline \multirow[t]{2}{*}{ PDSA registered } & No & $141(97 \%)$ & $409(93 \%)$ & (Reference level) & - \\
\hline & Yes & $5(3 \%)$ & $29(7 \%)$ & $0.50(0.19,1.32)$ & 0.161 \\
\hline \multirow[t]{2}{*}{ Osteoarthritis discussed } & No & $133(91 \%)$ & $431(98 \%)$ & (Reference level) & - \\
\hline & Yes & $13(9 \%)$ & $7(2 \%)$ & $6.02(2.35,15.39)$ & $<0.001$ \\
\hline \multirow[t]{2}{*}{ Lameness consultation } & No & $117(80 \%)$ & 391 (89 \%) & (Reference level) & - \\
\hline & Yes & $29(20 \%)$ & $47(11 \%)$ & $2.06(1.24,3.42)$ & 0.005 \\
\hline \multirow[t]{2}{*}{ Vomiting consultation } & No & $138(95 \%)$ & $402(92 \%)$ & (Reference level) & - \\
\hline & Yes & $8(5 \%)$ & $36(8 \%)$ & $0.65(0.29,1.43)$ & 0.281 \\
\hline \multirow[t]{2}{*}{ Diarrhoea consultation } & No & $143(98 \%)$ & $414(95 \%)$ & (Reference level) & - \\
\hline & Yes & $3(2 \%)$ & $24(5 \%)$ & $0.36(0.11,1.22)$ & 0.101 \\
\hline \multirow[t]{2}{*}{ Anal sacs expressed } & No & $139(95 \%)$ & $403(92 \%)$ & (Reference level) & - \\
\hline & Yes & $7(5 \%)$ & $35(8 \%)$ & $0.58(0.25,1.34)$ & 0.200 \\
\hline \multirow[t]{2}{*}{ Parasiticide prescribed $\dagger$} & No & $129(88 \%)$ & $390(89 \%)$ & (Reference level) & - \\
\hline & Yes & $17(12 \%)$ & $48(11 \%)$ & $1.07(0.59,1.93)$ & 0.820 \\
\hline \multirow[t]{2}{*}{ NSAID prescribed } & No & $45(69 \%)$ & $115(74 \%)$ & (Reference level) & - \\
\hline & Yes & $101(31 \%)$ & $323(26 \%)$ & $1.25(0.83,1.89)$ & 0.284 \\
\hline \multicolumn{6}{|l|}{ Multiple regression } \\
\hline \multicolumn{2}{|l|}{ Practice health scheme } & & & $5.35(1.57,18.17)$ & 0.007 \\
\hline \multicolumn{2}{|l|}{ Microchipped } & & & $0.43(0.41,0.91)$ & 0.015 \\
\hline Osteoarthritis discussed & & & & $5.42(2.09,14.07)$ & $<0.001$ \\
\hline Lameness consultation & & & & $2.02(1.20,3.42)$ & 0.008 \\
\hline
\end{tabular}

Frequencies and relative frequencies by case and control groups of dogs either positive or negative for each variable are displayed in the tabular format, with corresponding OR, $95 \% \mathrm{Cl}$ and $P$ values. JRT, Jack Russell terrier; SBT, Staffordshire bull terrier; WHWT, West Highland white terrier; PDSA, People's Dispensary for Sick Animals; NSAID, nonsteroidal anti-inflammatory drug.

${ }^{*}$ Age data expressed as median (range)

†Parasiticide medication prescribed during the consultation, and included endoparasitic and ectoparasitic drugs.

\section{Risk factors for recording overweight status}

With simple logistic regression analysis (Table 1), factors associated with a veterinarian recording of OW status included discussing osteoarthritis, discussing lameness, being microchipped and being a member of a practice health scheme. The same factors remained significant in the final multiple regression model. No other factors examined were of significance, either with simple or multiple regression.

\section{Discussion}

The present study has demonstrated that veterinarians in the first-opinion practice rarely record the OW status of dogs. In only $1.4 \%$ of consultations, did the contemporaneous free-text entry indicate that dogs were either OW or obese. These data are consistent with those of a previous study, which demonstrated that veterinarians rarely perform weight measurements and body condition scoring ${ }^{(12)}$. However, the present study expands upon the previous work because it was much larger (e.g. 49488 v. 148 dogs), the study population more representative, data were taken directly from the veterinary practice management software, and it asked a different research question: the previous study asked how frequently body weight is measured and a BCS is performed in primary care practice; in contrast, the present study asked how frequently veterinarians record the OW status of a dog with their owner, and what prompts 
them to do this. Both studies also used different research methodology. In the previous study, the computer records sent to a second-opinion veterinary hospital were reviewed for evidence of body weight measurements and BCS. In the present study, data were obtained as part of a national surveillance project, and directly collected from the practice management software. This enabled patient records to be examined for evidence of terms associated with the OW status. Despite these differences, both studies confirm that veterinarians infrequently assess the OW status in dogs that they examine.

Given that BCS was uncommonly recorded, it was not possible to determine the prevalence of OW status in the present population. However, if prevalence is similar to the expected prevalence in UK dogs, based on the recent studies ${ }^{(3,4)}$, this suggests that the condition is markedly underreported. All such findings have wide-reaching consequences for the veterinary profession, since they suggest that many dogs are not treated despite the well-recognised health consequences, which include increased disease risk ${ }^{(13)}$, shortened lifespan ${ }^{(14)}$, metabolic dysfunction $^{(15)}$ and decreased quality of life ${ }^{(16)}$. Interestingly, veterinarians have specified obesity as one of the main issues they could do more for as a profession ${ }^{(17)}$, indicating possible awareness of underdiagnosis. Finding the most appropriate means of communicating with owners regarding the topic of obesity ${ }^{(18,19)}$ might be a useful strategy in ensuring more veterinarians are prepared to actively engage owners with OW dogs.

Since only a few studies have examined veterinary decisionmaking for obesity, the reasons for the observed underreporting are not known. However, better understanding the reasons might allow strategies for change to be identified within the profession. One possible explanation for why the OW status was not recorded would be the time constraints encountered in general practice; in this respect, there might be other problems requiring more urgent attention. This is, perhaps, compounded by the fact owners rarely present their pet to the veterinarian because they are worried about excess body weight ${ }^{(6)}$, which itself might be related to the increased tendency for owners of OW dogs to underestimate their body condition, than owners of ideal weight $\operatorname{dogs}{ }^{(5-7)}$. In addition, veterinarians might be reluctant to record the OW status if they perceive that they will encounter resistance from owners, or be concerned about offending the owner. This reluctance might arguably be greater when owners are themselves OW, and it is noteworthy that a positive association between BMI in owners and the body condition of their dog exists ${ }^{(3,4)}$.

A second aim was to determine what factors were associated with the likelihood of a veterinarian recording OW status, and this was explored in the case-control part of the study. The OW status was more often recorded when dogs presented with either osteoarthritis or lameness, which is logical given their known association in this species ${ }^{(2,13)}$. It also emphasises that veterinarians more commonly highlight the OW status where they perceive either an association with a current health concern, or that weight management might benefit mobility. In contrast, there was no association between dispensing of NSAID and recording of OW status. While NSAID usage was common, being dispensed in $27 \%$ of all consultations, they were given for osteroarthritis and lameness in only a minority of cases (3 and 13\%, respectively). Therefore, the lack of association between NSAID use and recording the OW status might reasonably be explained by the fact that NSAID have a wide range of indications, including analgesia for non-orthopaedic problems and post-operative analgesia. Furthermore, NSAID were commonly dispensed during repeat prescription consultations and, in such circumstances, the OW status is unlikely to be recorded.

The OW status was also more commonly recorded when dogs were enrolled in a practice health scheme (a scheme whereby complimentary routine preventive health care and some additional veterinary fees are provided an upfront fee). This would be expected, since OW dogs might have joined such a scheme because they were OW. In addition, since such schemes focus on preventive health care, and would include discussions regarding maintaining a healthy weight, greater recording of the OW status would be expected. In contrast to this, the OW status was less commonly recorded in dogs that were listed on the practice system as microchipped; however, the reason for this association is unknown.

The study has a number of limitations, which should be considered. Firstly, it was retrospective in nature, and used data gathered from practice software; as a result, and some information is incomplete or missing. For instance, occasionally basic signalment data were missing, such that some consultations were excluded which might have been relevant. Secondly, the study's search terms mainly utilised the free text entered during the consultation. This free text quality most likely varied between practices and veterinarians, and might not have represented the actual extent of the discussions between veterinarian and client. Thirdly, the use of key words to identify consultations within this free text, particularly when identifying case animals was another limiting factor. Although an effort was made to consider all possible complete word, and shorthand terms for the OW status, some might have been missed. Thus, the results obtained might be an underestimate of the actual situation. That said, it is unlikely that this point alone would account for the discrepancy between the current prevalence of dogs being OW and obese, and the frequency of recording the OW status.

A fourth limitation was the method by which OW status was recorded for control dogs. Unfortunately, we were unable to use BCS to identify controls, because this parameter was rarely recorded, a finding consistent with other published work ${ }^{(12)}$. Such a problem is common to other studies of this nature ${ }^{(20)}$. Therefore, we chose to use bodyweight, stratified on breed, sex and neutered status, whereby dogs in the upper quartile were assumed to be OW. It is possible that this method incorrectly categorised some dogs as OW and vice versa.

Finally, there were limited breeds included in the present study due to excluding those without three matched controls and excluding all crossbreeds; results, therefore, cannot necessarily be applied to all breeds or to the crossbreed population. The three breeds of Labrador Retrievers, Jack Russell Terriers and Staffordshire Bull Terriers contributed proportionally the most consultations to the study. Owing to the Jack Russell Terrier not being recognised officially as a breed ${ }^{(21)}$, it appears overrepresented in the present study. Other than this, the breed spread is highly representative of the most commonly registered breeds ${ }^{(22)}$. We must also consider that, as dogs are often 
classified in the practice records based on the type they most represent and are not necessarily a pedigree of that breed, matching the breeds in the present study may not have overcome the actual variances in weight when calculating quartiles.

\section{Conclusion}

We conclude that there is a marked underreporting of OW status in pet dogs, but the reasons for this are not clear. Various factors were related to the likelihood of a veterinarian recording OW status, the most notable when the consultation was for osteoarthritis or lameness. While this suggests that veterinarians are aware of the association between the OW status and orthopaedic disease in dogs, lesser importance is placed on identifying obese dogs without such complications. This presents a challenge for strategies that aim to prevent the development of obesity, or to manage the condition when it arises. Further work is needed to understand the reasons behind decisionmaking in veterinarians, when faced with an OW or obese dog.

\section{Acknowledgements}

The authors would like to thank Small Animal Surveillance Network (SAVSNET) for providing the data to carry out the study. A. J. G.'s readership is financially supported by Royal Canin. This research received no specific grant from any funding agency, commercial or not-for-profit sectors. A. J. G.'s academic position at the University of Liverpool is financially supported by Royal Canin. The study was based upon a project conducted by N. C. R., as part of the requirements for the Degree of Bachelor of Veterinary Science. A. J. G. and P.-J. M. N. proposed the research question; P.-J. M. N. acquired the study data from the Small Animal Surveillance Network database; all authors assisted in study design; N. C. R. executed the study and analysed the data; N. C. R. produced the first draft of the manuscript, which was subsequently edited by both A. J. G. and P.-J. M. N. The work was performed at School of Veterinary Science, University of Liverpool, Leahurst Campus, Chester High Road, Neston, Wirral, CH64 7TE, UK.

This paper was published as part of the WALTHAM International Nutritional Sciences Symposium Proceedings 2013, publication of which was supported by an unrestricted educational grant from Mars Incorporated. The papers included in these proceedings were invited by the Guest Editor and have undergone the standard journal formal review process. They may be cited.

\section{Supplementary material}

The supplementary material for this article can be found at http://www.journals.cambridge.org/jns

\section{References}

1. Kopelman PG (2006) Obesity as a medical problem. Nature 404, 635-643.
2. German AJ (2006) The growing problem of obesity in dogs and cats. J Nutr 136, 1940S-1946S.

3. Holmes KL, Morris PJ, Abdulla Z, et al. (2007) Risk factors associated with excess body weight in dogs in the UK. J Anim Physiol Anim Nutr 91, 166-167.

4. Courcier EA, Thomson RM, Mellor DJ, et al. (2010) An epidemiological study of environmental factors associated with canine obesity. J Small Anim Pract 51, 362-367.

5. Courcier EA, Mellor DJ, Thomson RM, et al. (2011) A cross sectional study of the prevalence and risk factors for owner misperception of canine body shape in first opinion practice in Glasgow. Prev Vet Med 102, 66-74.

6. White GA, Cobb K, Hammond R, et al. (2011) Canine obesity: is there a difference between veterinarian and owner perception? J Small Anim Pract 52, 622-626.

7. Eastland-Jones R, German AJ, Holden SL, et al. (2013) Owner Misperception of Canine Body Condition Persists Despite Use of BCS Chart. (WINSS 2013 proceedings, P90 - add JNSref details later, if accepted).

8. Degeling C, Rock M \& Toews L (2011) Portrayals of canine obesity in English-language newspapers and in leading veterinary journals, 2000-2009: implications for animal welfare organizations and veterinarians as public educators. J Appl Anim Welf Sci 14, 286-303.

9. Shaw JR, Adams CL, Bonnett BN, et al. (2008) Veterinarian-clientpatient communication during wellness appointments versus appointments related to a health problem in companion animal practice. J Am Vet Med Assoc 233, 1576-1586.

10. Morris A (2009) Animal welfare: whose responsibility? Vet Rec 164, 705-707.

11. Coe JB, Adams CL \& Bonnett BN (2008) A focus group study of veterinarians' and pet owners' perceptions of veterinarian-client communication in companion animal practice. J Am Vet Med Assoc 233, 1072-1080.

12. German AJ \& Morgan LE (2008) How often do veterinarians assess the bodyweight and body condition of dogs? Vet Rec 163, 503-505.

13. Lund EM, Armstrong J, Kirk CA, et al. (2006) Prevalence and risk factors for obesity in adult dogs from private US veterinary practices. Int J Appl Res Vet Med 4, 177-186.

14. Kealy RD, Lawler DF, Ballam JM, et al. (2002) Effects of diet restriction on life span 481 and age-related changes in dogs. J Am Vet Med Assoc 220, 1315-1320.

15. Tvarijonaviciute A, Ceron JJ, Holden SL, et al. Obesity-related metabolic dysfunction in dogs: a comparison with human metabolic syndrome. BMC Vet Res 8, 147.

16. German AJ, Holden SL, Wiseman-Orr ML, et al. (2012) Quality of life is reduced in obese dogs but improves after successful weight loss. Vet J 192, 428-434.

17. Yeates JW \& Main DC (2011) Veterinary surgeons' opinions on dog welfare issues. J Small Anim Pract 52, 464-468.

18. Wheat H, McMartin C \& Coe JB (2013) Veterinarian-initiated longterm dietary recommendations: practitioners' management of clients' responses. WINSS 2013 Proceedings P70. - use JNS publication ref?

19. Wheat H, McMartin C \& Coe JB (2013) The importance of question design in maximizing nutritional history-taking. WINSS 2013 Proceedings P71. - use JNS publication ref?

20. Carina S \& Morris P (2013) Associations between longevity and body condition in domestic dogs. WINSS 2013 Proceedings P52- use JNS publication ref?

21. The Kennel Club (2013) Breed Information Centre - Parson Russell Terrier (2013). Available at http://www.the-kennel-club.org.uk/ services/public/breed/display.aspx?id=3175 (accessed May 2013).

22. Asher L, Buckland EL, Phylactopoulos CI, et al. (2011) Estimation of the number and demographics of companion dogs in the UK. BMC Vet Res 7, 74. 